\title{
The effect of incomplete mixing upon quadratic autocatalysis
}

\author{
A. H. Msmali ${ }^{1} \quad$ M. I. Nelson ${ }^{2} \quad$ M. Edwards ${ }^{3}$
}

(Received 9 January 2012; revised 23 May 2012)

\begin{abstract}
We analyse a model for a continuously stirred tank reactor with imperfect mixing in which the reactor is represented by two well mixed compartments with material transfer between them. These reactors represent 'highly agitated' and 'less agitated' regions. The chemical model used is a quadratic autocatalytic scheme with linear decay of the autocatalyst. We investigate how the reactor performance depends upon the degree of mixing in the reactor and the size of the less agitated region. Surprisingly, the performance of the reactor with sufficiently small values of mixing is inferior to that with no mixing between the compartments.
\end{abstract}

http://journal.austms.org.au/ojs/index.php/ANZIAMJ/article/view/5105 gives this article, (c) Austral. Mathematical Soc. 2012. Published June 19, 2012. ISSN 1446-8735. (Print two pages per sheet of paper.) Copies of this article must not be made otherwise available on the internet; instead link directly to this URL for this article. 


\section{Contents}

1 Introduction

C267

2 Model equations

C268

2.1 Dimensional mathematical models . . . . . . . . . . . C269

2.2 Dimensionless model . . . . . . . . . . . . . . . . . . C269

3 Steady state solution branches

$\mathrm{C} 270$

4 Stability of the washout branch

C271

5 Results

$\mathrm{C} 272$

5.1 Steady state diagram: reactant concentration . . . . . . C272

5.2 Steady state diagram: autocatalytic concentration . . . . .

$\mathrm{C} 275$

6 Conclusions

$\mathrm{C} 277$

References

C279

\section{Introduction}

In many reaction engineering models the reactor contents are assumed to be perfectly mixed. This assumption may be good for small reactors but incomplete mixing becomes more likely as the size of the reactor increases. We investigate the effect that incomplete mixing has by employing a two parameter mixing model in which a non-ideal reactor is represented by two well mixed compartments with mass transfer between them. One of these compartments, near the impeller, is 'highly agitated' whilst the other is 'less agitated' [2, Chapter 14.3.2]. For small values of the mixing parameter $\delta$ the latter represents a stagnant region. This two parameter model was first proposed by Corrigan and Beavers [1]. This mixing model is illustrated in Figure 1. 


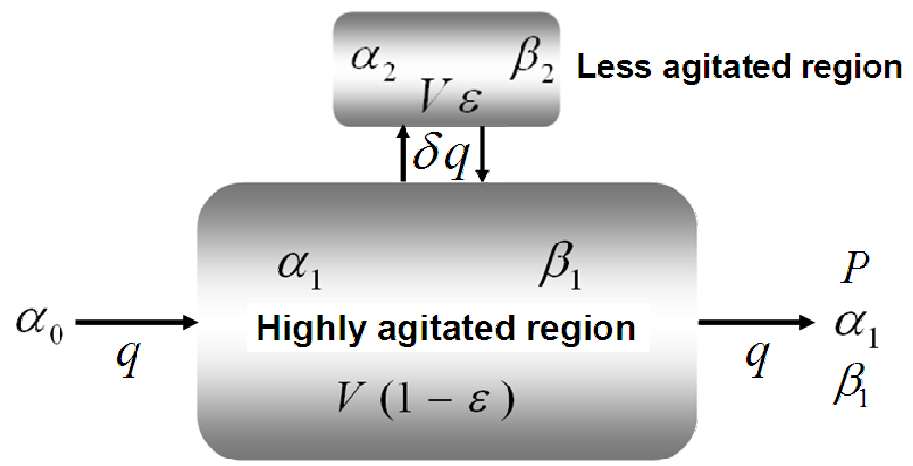

Figure 1: Schematic diagram for a reactor assuming non-ideal mixing.

\section{$2 \quad$ Model equations}

The chemical reaction model is

$$
\begin{aligned}
& \mathrm{A}+\mathrm{B} \rightarrow 2 \mathrm{~B} \quad \text { rate }=\mathrm{k}_{1} \mathrm{ab}, \quad \text { quadratic autocatalytic reaction, } \\
& \mathrm{B} \rightarrow \mathrm{C} \quad \text { rate }=\mathrm{k}_{2} \mathrm{~b}, \quad \text { decay of autocatalyst. }
\end{aligned}
$$

Quadratic autocatalysis is a simple prototype for more complicated kinetic structures possessed by large families of enzyme systems and surface catalysis [3]. 


\subsection{Dimensional mathematical models}

The dimensional model equations for the case of imperfect mixing are

$$
\begin{aligned}
V(1-\varepsilon) \frac{d a_{1}}{d t}= & q\left(a_{0}-a_{1}\right)-V(1-\varepsilon) k_{1} a_{1} b_{1}-q \delta\left(a_{1}-a_{2}\right) \\
V(1-\varepsilon) \frac{d b_{1}}{d t}= & q\left(b_{0}-b_{1}\right)+V(1-\varepsilon) k_{1} a_{1} b_{1} \\
& -V(1-\varepsilon) k_{2} b_{1}-q \delta\left(b_{1}-b_{2}\right), \\
V \varepsilon \frac{d a_{2}}{d t}= & q \delta\left(a_{1}-a_{2}\right)-V \varepsilon k_{1} a_{2} b_{2}, \\
V \varepsilon \frac{d b_{2}}{d t}= & q \delta\left(b_{1}-b_{2}\right)+V \varepsilon k_{1} a_{2} b_{2}-V \varepsilon k_{2} b_{2} .
\end{aligned}
$$

The mixing parameters are $\varepsilon$, the size of the less agitated region, and $\delta$, the strength of the mixing.

\subsection{Dimensionless model}

To non-dimensionalise Equations (1)-(4) we introduce the variables groups $\alpha_{i}=a_{i} / a_{0}, \beta_{i}=b_{i} / a_{0}$ and $t^{*}=k_{1} a_{0} t$. System (1)-(4) then becomes

$$
\begin{aligned}
& \frac{d \alpha_{1}}{d t^{*}}=\frac{1-\alpha_{1}}{(1-\varepsilon) \tau^{*}}-\alpha_{1} \beta_{1}-\frac{\delta\left(\alpha_{1}-\alpha_{2}\right)}{(1-\varepsilon) \tau^{*}}, \\
& \frac{d \beta_{1}}{d t^{*}}=\frac{\beta_{0}-\beta_{1}}{(1-\varepsilon) \tau^{*}}+\alpha_{1} \beta_{1}-\kappa_{2} \beta_{1}-\frac{\delta\left(\beta_{1}-\beta_{2}\right)}{(1-\varepsilon) \tau^{*}}, \\
& \frac{d \alpha_{2}}{d t^{*}}=\frac{\delta\left(\alpha_{1}-\alpha_{2}\right)}{\varepsilon \tau^{*}}-\alpha_{2} \beta_{2}, \\
& \frac{d \beta_{2}}{d t^{*}}=\frac{\delta\left(\beta_{1}-\beta_{2}\right)}{\varepsilon \tau^{*}}+\alpha_{2} \beta_{2}-\kappa_{2} \beta_{2} .
\end{aligned}
$$

The non-dimensional parameter groups are: the scaled concentration of autocatalyst in the feed, $\beta_{0}=b_{0} / a_{0}$; the scaled decay rate, $k_{2}=k_{2} /\left(k_{1} a_{0}^{2}\right)$ and the scaled residence time $\tau^{*}=\left(\mathrm{Vk}_{2} \mathrm{a}_{0}{ }^{2}\right) / \mathrm{q}$. From now we assume that there is no autocatalyst in the feed, that is, $b_{0}=\beta_{0}=0$ and that $\kappa_{2}<1$. 


\section{Steady state solution branches}

The system (5)-(8) has two steady state solution branches. The washout solution is

$$
\left(\alpha_{1, w}, \beta_{1, w}, \alpha_{2, w}, \beta_{2, w}\right)=(1,0,1,0) .
$$

The no-washout solution is

$$
\left(\alpha_{1, n w}, \beta_{1, n w}, \alpha_{2, n w}, \beta_{2, n w}\right)=\left(\hat{\alpha}_{1}, \hat{\beta}_{1}, \hat{\alpha}_{2}, \hat{\beta}_{2}\right)
$$

where

$$
\begin{aligned}
& \hat{\beta}_{1}=\frac{\delta \hat{\beta}_{2}}{1+\delta+\left(\kappa_{2}-\hat{\alpha}_{1}\right)(1-\varepsilon) \tau^{*}}, \quad \hat{\alpha}_{2}=\frac{\delta \alpha_{1}}{\left(\delta+\beta_{2} \varepsilon \tau^{*}\right)}, \\
& \hat{\beta}_{2}=\frac{\delta\left[-\left(\hat{\alpha}_{1}-\kappa_{2}\right)^{2}(1-\varepsilon) \varepsilon \tau^{* 2}+(\varepsilon+\delta)\left(\hat{\alpha}_{1}-\kappa_{2}\right) \tau^{*}-\delta\right]}{\tau^{*} \varepsilon\left\{-(1-\varepsilon)\left(\hat{\alpha}_{1}-\kappa_{2}\right) \varepsilon \kappa_{2} \tau^{* 2}-\left[(1-\varepsilon) \delta \alpha_{1}-\kappa_{2}(\varepsilon+\delta)\right] \tau^{*}+\delta\right\}} .
\end{aligned}
$$

Using Maple, we obtain a cubic equation for $\alpha_{1}$

$$
G\left(\hat{\alpha}_{1}\right)=A_{3} \tau^{* 3} \hat{\alpha}_{1}^{3}+A_{2} \tau^{* 2} \hat{\alpha}_{1}^{2}+A_{1} \tau^{*} \hat{\alpha}_{1}+A_{0},
$$

where the coefficients $A_{i}$ are

$$
\begin{aligned}
A_{3}= & (1-\varepsilon)^{2} \varepsilon\left[\tau^{*} \varepsilon \kappa_{2}(1+\delta)+\delta\right], \\
A_{2}= & -(1-\varepsilon) \varepsilon\left\{\left(2 \kappa_{2}+3 \kappa_{2} \delta+1\right)(1-\varepsilon) \varepsilon \kappa_{2} \tau^{* 2}\right. \\
& \left.+\left[(1-\varepsilon) \delta+2(1+\delta) \kappa_{2}(\varepsilon+\delta)\right] \tau^{*}+2 \delta(1+\delta)\right\}, \\
A_{1}= & \varepsilon^{2} \kappa_{2}^{2}(1-\varepsilon)^{2}\left(2+\kappa_{2}+3 \kappa_{2} \delta\right) \tau^{* 3} \\
& +\varepsilon \kappa_{2}(1-\varepsilon)\left\{5 \varepsilon \kappa_{2} \delta+2 \varepsilon\left(1+\kappa_{2}\right)+\delta\left[\kappa_{2}(1+4 \delta)+2\right]\right\} \tau^{* 2} \\
& +\left\{\delta \varepsilon(1-\varepsilon)(\delta+2)+\left[\delta^{3}+\varepsilon\left(3(2-\varepsilon) \delta^{2}+(\varepsilon+2) \delta+\varepsilon\right)\right] \kappa_{2}\right\} \tau^{*} \\
& +\delta\left(\delta^{2}+\varepsilon+2 \delta \varepsilon\right), \\
A_{0}= & -\left\{\delta \varepsilon \tau^{* 2}(1-\varepsilon) \kappa_{2}^{2}+\left[\delta(\varepsilon+\delta)+\tau^{*} \varepsilon(1-\varepsilon)\right] \tau^{*} \kappa_{2}+\varepsilon \tau^{*}(1+\delta)+\delta^{2}\right\} \\
& \times\left\{\delta+\left[(1-\varepsilon) \varepsilon \kappa_{2} \tau^{*}+\varepsilon+\delta\right] \tau^{*} \kappa_{2}\right\} .
\end{aligned}
$$


We have $A_{3}>0, A_{2}<0, A_{1}>0$ and $A_{0}<0$. As there are three sign changes in the sequence of coefficients of $G\left(\hat{\alpha}_{1}\right)$ there are either one or three real positive roots (Descarte's Rule of Signs [4, Appendix A 2.2]). Calculation shows that

$$
\begin{aligned}
\mathrm{G}\left(\kappa_{2}\right)= & -\varepsilon^{2}(1+\delta)^{2}-\left(1-\kappa_{2}\right) \kappa_{2} \tau^{* 2} \\
& -\left[\varepsilon\left(1+\delta-\kappa_{2}\right)+\kappa_{2} \delta^{2}\right] \delta \tau^{*}-\delta^{3}<0,
\end{aligned}
$$

(as $\left.0<\kappa_{2}<1\right)$ and $G\left(\frac{1+\delta}{(1-\varepsilon) \tau^{*}}+\kappa_{2}\right)=\delta^{4}\left[1+\tau^{*} \kappa_{2}(1-\varepsilon)\right]>0$. Hence there is always a solution of Equation (11) with

$$
\kappa_{2}<\hat{\alpha}_{1}<\kappa_{2}+\frac{1+\delta}{(1-\varepsilon) \tau^{*}} .
$$

Based on numerical experimentation we conjecture that when the no-washout solution is physically meaningful it is uniquely defined.

\section{Stability of the washout branch}

The stability of the steady state solutions for the system composing Equations (5)-(8) are determined by the eigenvalues of the Jacobian matrix evaluated at the steady state solution. A steady state solution is stable if the real part of all eigenvalues are less than zero.

Evaluating the Jacobian matrix along the washout branch we obtain

$$
J(1,0,1,0)=\left[\begin{array}{cccc}
-\frac{(1+\delta)}{(1-\varepsilon) \tau^{*}} & -1 & \frac{\delta}{(1-\varepsilon) \tau} & 0 \\
0 & -\frac{(1+\delta)}{(1-\varepsilon) \tau^{*}}+1-\kappa_{2} & 0 & \frac{\delta}{(1-\varepsilon) \tau^{*}} \\
\frac{\delta}{\varepsilon \tau^{*}} & 0 & -\frac{\delta}{\varepsilon \tau^{*}} & -1 \\
0 & \frac{\delta}{\varepsilon \tau^{*}} & 0 & -\frac{\delta}{\varepsilon \tau^{*}}+1-\kappa_{2}
\end{array}\right] .
$$

After some algebra we find that the washout solution is stable when

$$
\tau^{*}<\tau_{\mathrm{cr}}^{*}=\frac{1}{1-\kappa_{2}} \times \frac{\delta+\varepsilon-\sqrt{(\delta-\varepsilon)^{2}+4 \delta \varepsilon^{2}}}{2 \varepsilon(1-\varepsilon)} .
$$


The washout branch is unstable if $\tau^{*}>\tau_{\mathrm{cr}}^{*}$. We conjecture that when the washout solution is stable the no-washout solution is not physically meaningful; and when the washout solution is unstable the washout solution is stable.

\section{$5 \quad$ Results}

We used Maple to find the physical meaningful steady state solutions of system and determine their stability.

\subsection{Steady state diagram: reactant concentration}

In this section we consider the steady state diagram for the dimensionless reactant concentration $\left(\alpha_{1}\right)$ in ideal and non-ideal reactors. The application we have in mind is the use of a bioreactor to remove a pollutant $\left(\alpha_{1}\right)$.

Figure 2 shows the steady state curve for the dimensionless reactant concentration for ideal and non-ideal reactors as a function of the dimensionless residence time. The critical value of the dimensionless residence time for the ideal reactor (1.03) is higher than that in the non-ideal reactor (0.52). Below these values only the washout branch is stable and process failure occurs.

The two solution curves intersect when $\tau^{*}=1.4$. Over the range $0.52<\tau^{*}<$ 1.4 the reactant concentration in the non-ideal reactor is lower than that in the ideal reactor, that is, the performance of the non-ideal reactor is superior to that of the ideal reactor. If $\tau^{*}>1.4$, then the reactant concentration in the ideal reactor is lower than that in the non-ideal reactor, that is, the performance of the ideal reactor is superior to that the non-ideal reactor.

We now suppose that it is desirable to reduce the concentration of pollutant in the effluent stream to $90 \%$ of its value in the influent stream. We denote the required residence time to achieve this by $\tau_{0.1}^{*}$. In Figure 2 the values are 13.89 and 21.4 for the idea and non-ideal mixing reactors respectively. 


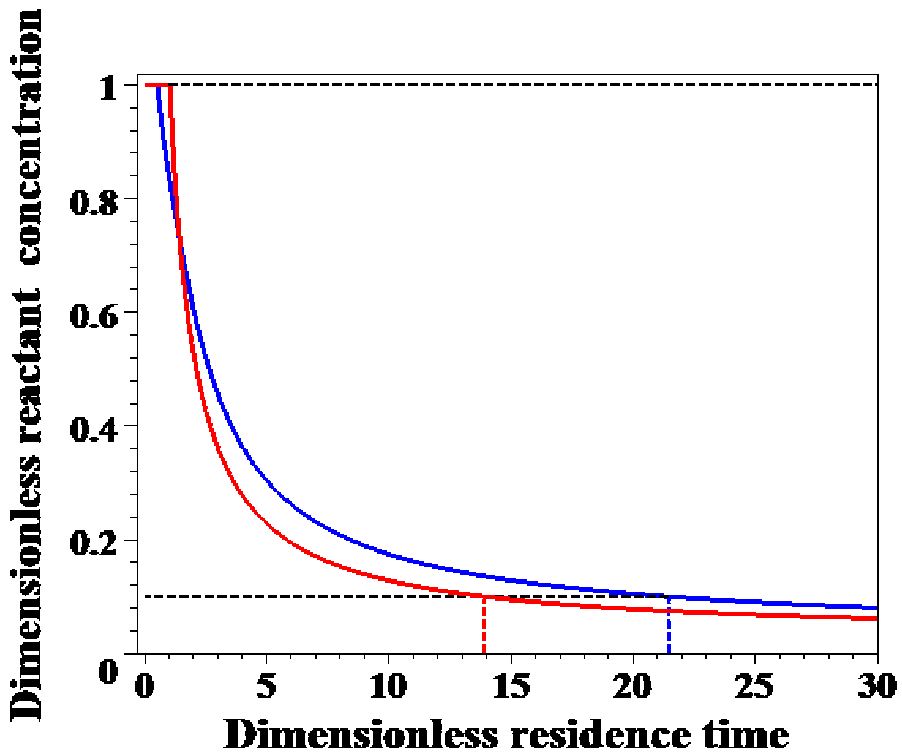

Figure 2: Steady state diagram for the dimensionless reactant concentration for ideal (red line) and non-ideal (blue line) mixing reactor as a function of the dimensionless residence time. Parameters are: $\kappa_{2}=0.028, \delta=\infty$ and $\varepsilon=0$ ideal; $\delta=0.2$ and $\varepsilon=0.3$ (non-ideal).

Figure 3 shows how the value of $\tau_{0.1}^{*}$ varies as a function of the mixing parameter for different sizes of the less agitated region.

As the mixing parameter increases towards infinity (ideal mixing) there is little difference between the curves. However, the figure has a surprising feature: when the mixing parameter is sufficiently small there is a region where the value of $\tau_{0.1}^{*}$ is higher than when there is no mixing.

In Table 1 we characterise the worst possible performance of a reactor, used to degrade the reactant species, at fixed $\varepsilon$, by a reactor index:

$$
\text { R-I }=100 \times \frac{\tau_{0.1}^{*}\left(\delta=\delta_{\max }\right)-\tau_{0.1}^{*}(\delta=\infty)}{\tau_{0.1}^{*}(\delta=\infty)}
$$




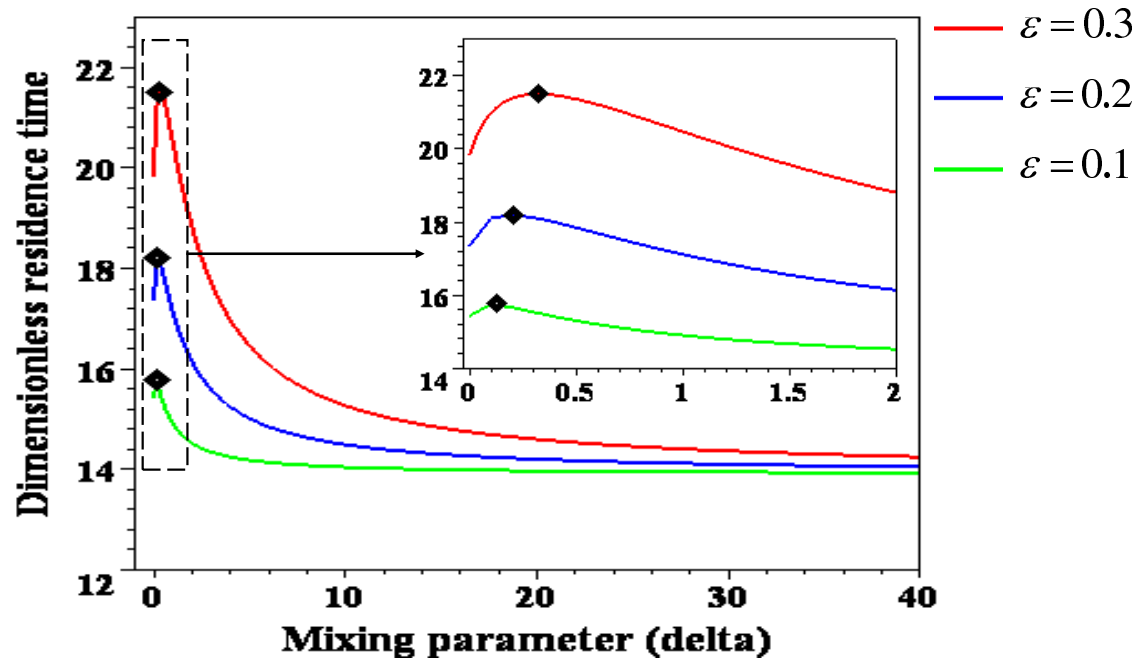

Figure 3: Dimensionless residence time $\left(\tau_{0.1}^{*}\right)$ as a function of the mixing parameter for three values of the less agitated region $(\varepsilon)$. Parameters $\kappa_{2}=$ $0.028 \varepsilon=0.3$ (red line), $\varepsilon=0.2$ (blue line) and $\varepsilon=0.1$ (green line).

Table 1: Reactor index calculation for $\tau_{0.1}^{*}$. The value of $\tau_{0.1}^{*}(\delta=\infty)$ is 13.889 . Parameter $\kappa_{2}=0.028$.

\begin{tabular}{lcccc}
\hline$\varepsilon$ & 0.1 & 0.2 & 0.3 & 0.4 \\
\hline$\delta_{\max }$ & 0.127 & 0.205 & 0.328 & 0.500 \\
$\tau_{0.1}^{*}\left(\delta_{\max }\right)$ & 15.768 & 18.94 & 21.51 & 26.23 \\
$\mathrm{R}-\mathrm{I}$ & 13.53 & 36.37 & 54.87 & 88.85 \\
\hline
\end{tabular}

For example, when $\varepsilon=0.1$ the maximum increase in the value of the parameter $\tau_{0.1}^{*}$ for a reactor with incomplete mixing is $14 \%$ more than the value for a reactor with perfect mixing.

Figure 4 shows that as the size of the less agitated region increases the reactor index increases. For larger values of $\varepsilon$ the reactor index increases sharply. 


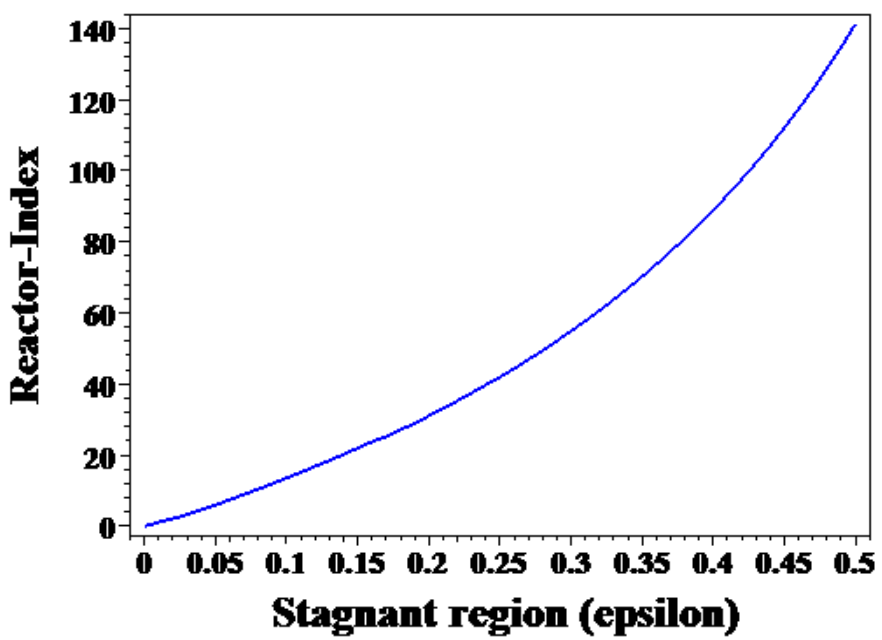

Figure 4: Reactor index for the parameter $\tau_{0.1}^{*}$ as a function of the less agitated region $(\varepsilon)$. Parameter $\kappa_{2}=0.028$.

\subsection{Steady state diagram: autocatalytic concentration}

Here we consider the steady state diagram for the dimensionless autocatalytic concentration $\left(\beta_{1}\right)$ in ideal and non-ideal reactors. The application we have in mind is the use of a bioreactor to produce the autocatalytic species $\left(\beta_{1}\right)$.

Figure 5 shows the steady state diagram for the dimensionless autocatalytic concentration for ideal and non-ideal reactors. In both cases process failure occurs, that is, the autocatalyst concentration is zero if the residence time is below a critical value. The maximum value of the autocatalyst concentration $\left(\beta_{1, \max }\right)$ is 0.69 and 0.65 for ideal and non-ideal reactors respectively. The corresponding values of the residence time are 7.18 and 9.29 respectively. Incomplete mixing has a greater effect upon the required value of the residence time rather than the maximum value. 


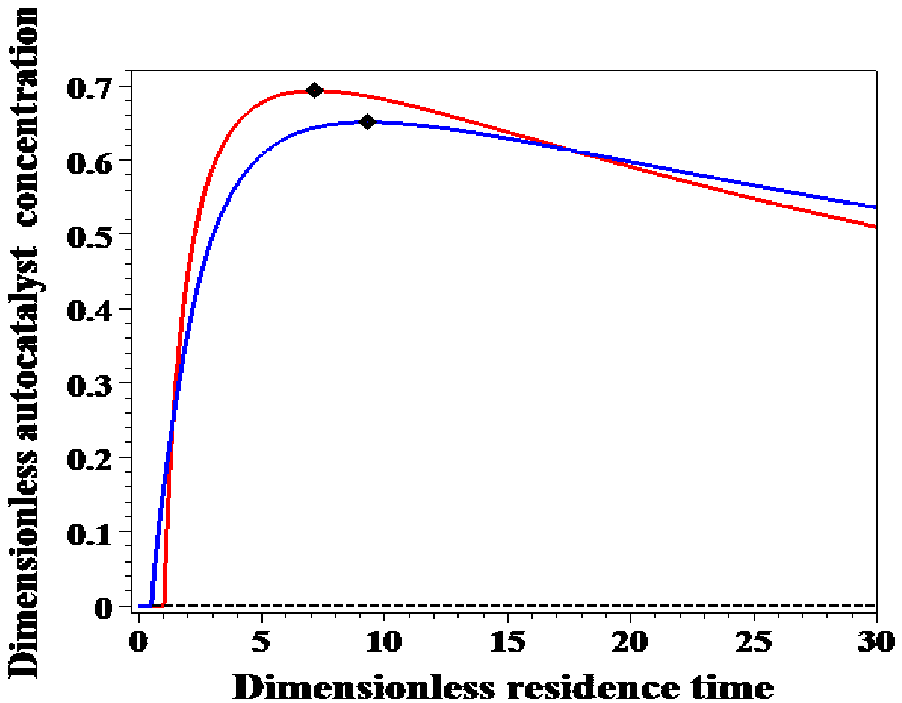

Figure 5: Steady state diagram for the dimensionless autocatalytic concentration for ideal (red line) and non-ideal (blue line) reactors as a function of the dimensionless residence time. Parameters are: $\kappa_{2}=0.028, \delta=\infty$ and $\varepsilon=0$ ideal; $\delta=0.2$ and $\varepsilon=0.3$ (non-ideal).

Figure 6 shows the maximum autocatalytic concentration $\left(\beta_{1, \max }\right)$ as a function of the mixing parameter $(\delta)$ for three sizes of the less agitated region $(\varepsilon)$. As the mixing parameter increases towards infinity the values converge to the perfectly mixed values. However, the figure shows a surprising feature: when the mixing parameter is sufficiently small there is a 'poor mixing' region in which the value of $\beta_{1, \max }$ is smaller than when there is no mixing.

In Table 2 we characterise the worst possible performance of a reactor used to produce the autocatalytic species, at fixed $\varepsilon$, by the reactor index

$$
\mathrm{R}-\mathrm{I}=100 \times \frac{\beta_{1, \max }\left(\delta=\delta_{\min }\right)-\beta_{1, \max }(\delta=\infty)}{\beta_{1, \max }(\delta=\infty)} .
$$

This table shows, for example, that when $\varepsilon=0.1$ the maximum decrease in the value of the parameter $\beta_{1, \max }^{*}$ is $1.7 \%$ more than the value for a reactor 


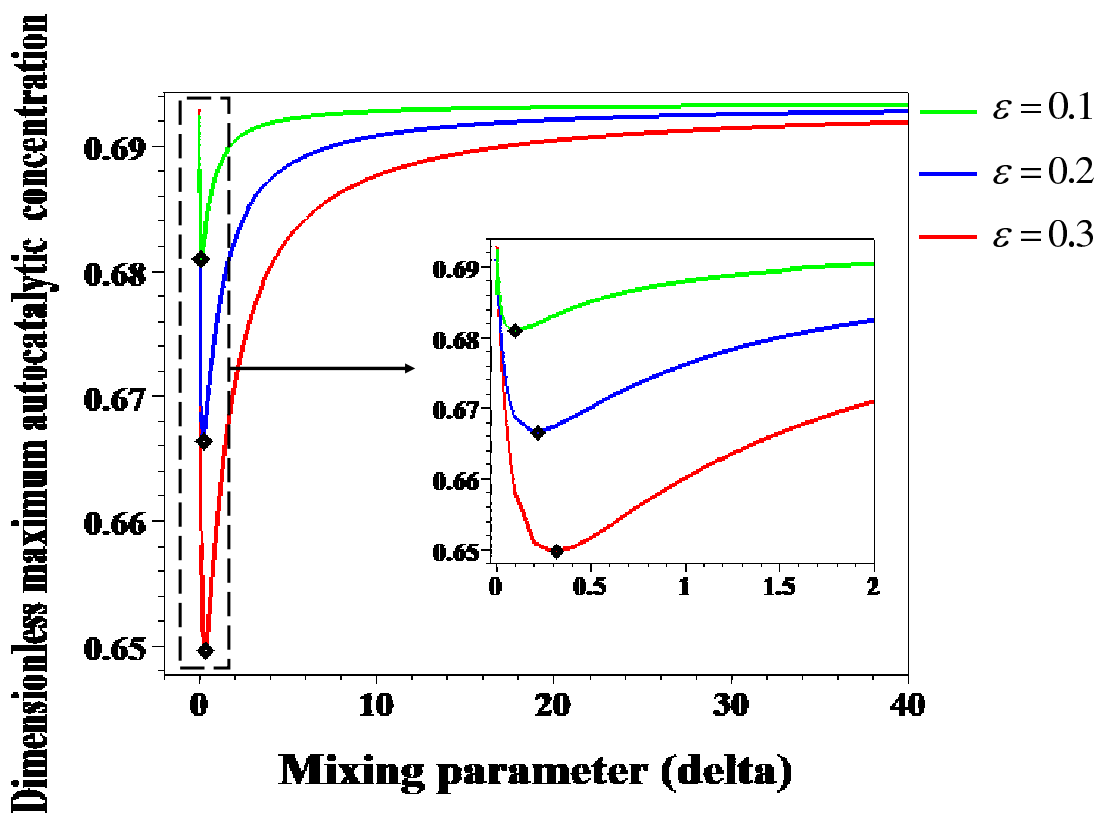

Figure 6: The maximum autocatalytic concentration value $\left(\beta_{1, \max }\right)$ as a function of the mixing parameter for three values of the less agitated region $(\varepsilon)$. Parameter $\kappa_{2}=0.028$.

with perfect mixing. We note that although incomplete mixing has little effect on the maximum concentration that can be obtained, it will have a much greater effect on the productivity which is defined by $\operatorname{Pr}=\beta_{1} / \tau$.

\section{Conclusions}

We investigated the effect of incomplete mixing upon the performance of a chemical reactor. The reaction scheme used was quadratic autocatalysis with decay of the autocatalyst species. A two parameter mixing model was used in which the reactor is divided into two compartments comprising highly 
Table 2: Reactor index calculation for $\beta_{1, \max }$. The value of $\beta_{1, \min }(\delta=\infty)$ is 0.693 . Parameter $\kappa_{2}=0.028$.

\begin{tabular}{lcccc}
\hline$\varepsilon$ & 0.1 & 0.2 & 0.3 & 0.4 \\
\hline$\delta_{\min }$ & 0.104 & 0.221 & 0.316 & 0.413 \\
$\tau_{\max }^{*}\left(\delta_{\min }\right)$ & 07.68 & 08.17 & 08.83 & 09.70 \\
$\min \left(\beta_{1, \max }\right)$ & 0.681 & 0.666 & 0.650 & 0.629 \\
$\mathrm{R}-\mathrm{I}$ & -1.73 & -3.90 & -6.20 & -9.24 \\
\hline
\end{tabular}

agitated and less agitated regions. Mixing occurs between these regions.

We considered scenarios in which the reactor is either used to remove a pollutant or to produce the autocatalyst. In the former the reactor performance is characterised by the value of the residence time required to reduce the reactant concentration to $90 \%$ of its value in the feed. In the latter the performance is characterised by the maximum concentration of the autocatalyst. For sufficiently large values of the mixing parameter the behaviour of the incompletely mixed reactor approaches that of a well mixed reactor.

In both cases the effect of incomplete mixing was investigated through the use of an appropriate reactor index which characterises, for a fixed size of the less agitated region $(\varepsilon)$, the worst possible performance of the reactor. In both cases an interesting feature was identified, namely, for very small values of the mixing parameter the performance of the reactor is inferior to that of a reactor with no mixing between the two compartments.

Acknowledgements Ahmed Msmali gratefully acknowledges Umm AlQura University for a PhD scholarship. Financial support to attend EMAC 2011 from the CSIRO/ANZIAM Student Support Scheme is also acknowledged. 


\section{References}

[1] T. E. Corrigan and W. O. Beavers. Dead space interaction in continuous stirred tank reactors. Chemical Engineering Science, 23:1003-1006, 1968. doi:10.1016/0009-2509(83)80132-8 C267

[2] H. S. Fogler. Elements of Chemical Reaction Engineering. Prentice Hall PTR, Upper Saddle River, New Jersey, USA, third edition, 1999. C267

[3] P. Gray and S. K. Scott. Autocatalytic reactions in the isothermal continuous stirred tank reactor. Chemical Engineering Science, 38, 1983, 29-43. doi:10.1016/0009-2509(83)80132-8 C268

[4] J. D. Murray. Mathematical Biology, 2nd edition, 1989. C271

\section{Author addresses}

1. A. H. Msmali, School of Mathematics and Applied Statistics, University of Wollongong, New South Wales 2020, Australia. mailto: ahom8210uow.edu. au

2. M. I. Nelson, School of Mathematics and Applied Statistics, University of Wollongong, New South Wales 2020, AUSTRALIA. mailto:nelsonm@member.ams . org

3. M. Edwards, School of Mathematics and Applied Statistics, University of Wollongong, New South Wales 2020, Australia. mailto:maureen_edwards@uow.edu.au 